\title{
Threshold enhancement phenomena in $Y(4260)$ decays
}

\author{
Xiao-Hai Liu \\ Department of Physics and State Key Laboratory of Nuclear Physics \\ and Technology, Peking University, Beijing 100871, China \\ liuxiaohai@pku.edu.cn
}

Published 16 April 2014

\begin{abstract}
We investigate several strong and radiative decay modes of $Y(4260)$, by assuming that $Y(4260)$ either is a $D_{1} \bar{D}$ molecular state, or has sizeable couplings with $D_{0} \bar{D}^{*}$ and $D_{1}^{\prime} \bar{D}$. In such ansatzes, obvious threshold enhancements or narrow cusp structures appear quite naturally without introducing a genuine resonance. And we emphasize that the radiative decay modes may be useful for studying $D^{(*)} \bar{D} S$-wave scattering.
\end{abstract}

Keywords: $Y(4260)$; strong and radiative decays; threshold enhancements.

PACS Numbers: 13.25.Gv, 14.40.Pq, 13.75.Lb

\section{Introduction}

The charmonium-like state $Y(4260)$ was first observed by the BaBar collaboration in 2005. ${ }^{1}$ It was then confirmed by CLEO $^{2}$ and Belle. ${ }^{3}$ There is no direct correspondence of $Y(4260)$ in naive quark model classifications. Furthermore, as a charmonium candidate, it has not been observed in the hidden charm decay channels. ${ }^{4-8}$ The $R$-value scan at BES around $4.26 \mathrm{GeV}$ appears to have a dip instead of a bump structure. ${ }^{9}$ These peculiar characterristics initiate many discussions about the nature of $Y(4260)$, such as the explanations of hybrid, tetraquark state, $\chi_{c 0} \rho$ or $\chi_{c 1} \omega$ molecular state, conventional $c \bar{c}$ state renormalized by $\chi_{c 0} \omega, D_{1} \bar{D}$ or $D_{0} \bar{D}^{*}$ molecular state, and so on. The recent observation of BESIII revives the discussion on the nature of $Y(4260)$. A charged charmonium-like resonance structure $Z_{c}(3900)$ is observed in the invariant mass spectrum of $J / \psi \pi^{ \pm}$from $Y(4260) \rightarrow J / \psi \pi^{+} \pi^{-} .^{10}$ If the observed structure is a genuine particle, it obviously cannot be a conventional $c \bar{c}$ state. Now there exist two unusual states in this decay channel. This experimental result was later confirmed by Belle. ${ }^{11}$ And a similar $Z_{c}$ was also announced by CLEO in $\psi(4170) \rightarrow J / \psi \pi^{+} \pi^{-} .{ }^{12}$ Together with the observations of $Z(4430)$ in

This is an Open Access article published by World Scientific Publishing Company. It is distributed under the terms of the Creative Commons Attribution 3.0 (CC-BY) License. Further distribution of this work is permitted, provided the original work is properly cited. 
$\psi^{\prime} \pi^{ \pm},{ }^{13} Z_{1,2}$ in $\chi_{c 1} \pi^{ \pm},{ }^{14}$ and $Z_{b}$ in $\Upsilon(n S) \pi^{ \pm}\left(h_{b}(m P) \pi^{ \pm}\right),{ }^{15}$ these unusual charged states not only enrich our knowledge about hadron spectroscopy but also bring new challenges.

One remarkable characteristic of these unconventional charmonium-like states is that many of them are observed at the thresholds of some charmed anti-charmed meson pairs. Therefore, to some extent, it is reasonable to interpret them as molecular states. Another similar description is the coupled channel effect, which will play a role when investigating the pertinent hadron spectrum. Especially, the coupled channel effect might largely affect the threshold phenomena, which has ever been taken as a dynamical mechanism in explaining the observation of the charged botomonium-like (charmonium-like) structures. ${ }^{16-21}$ In this work, we will assume that $Y(4260)$ is either a $D_{1} \bar{D}$ molecular state, or has sizeable couplings with $D_{0} \bar{D}^{*}$ and $D_{1}^{\prime} \bar{D}$. Here $D_{1}$ and $D_{1}^{\prime}$ refer to the narrow and broad axial vector charmed mesons, i.e. $D_{1}(2420)(\Gamma \simeq 27 \mathrm{MeV})$ and $D_{1}(2430)(\Gamma \simeq 384 \mathrm{MeV})$, respectively. Under such assumptions, a consistent description of many of the experimental observations can be obtained, such as its non-obersvation in open charm decays, and the observation of $Z_{c}(3900) .{ }^{16,22,23}$ We will mainly concentrate on the discussion of the threshold phenomena in several strong and radiative decay channels of $Y(4260)$ under such assumptions.

\section{Threshold Enhancement Phenomena}

If $Y(4260)$ is a $D_{1} \bar{D}+$ c.c. molecular state, its main decay channel would be $\bar{D} D^{*} \pi+$ c.c. (the charge conjugate part c.c. will be implicit sometimes for brevity), as illustrated in Fig. 1(a). Since the momenta of the produced $\bar{D} D^{*}$ will be very small, the final state interactions (FSIs) can be expected to play a role when analyzing some decay processes. We illustrate the rescattering processes in Fig. 1(b), where A and B are some specified final particles, and the black bubble indicates some unknown couplings. If FSIs are strong, they may significantly change the decay properties. Some non-analytical structures of the transition amplitude such as the cusp effect may occur. We will explore several pertinent decay modes and discuss some interesting threshold behaviors in the following sections.

The transition amplitude will be estimated according to the heavy hadron chiral perturbation theory (HHChPT). The explicit formulae and details can be found in

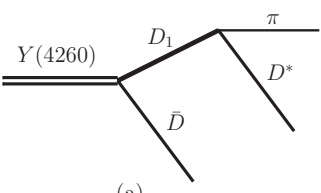

(a)



(b)

Fig. 1. (a): Diagram of the $Y(4260)$ main decay mode by supposing it is a $D_{1} \bar{D}$ molecular state. (b): The rescattering process, where A and B are some specified final states. The charge conjugate diagrams are implicit. 
Ref. 22. For the coupling of $Y(4260)$ with $D_{1} \bar{D}$, the pertinent effective Lagrangian is constructed as

$$
\mathcal{L}_{Y}=g_{Y} Y_{\mu}\left(D_{1}^{\mu} \bar{D}-\bar{D}_{1}^{\mu} D\right)
$$

which is an $S$-wave coupling.

Concerning the rescattering process illustrated in Fig. 1(b), we will discuss an interesting singularity that may appear in the transition amplitude, i.e., with some special kinematic configurations, all of the three intermediate states contained in the loop can be on-shell simultaneously. This is the so called triangle singularity (TS) or "two cut" condition. ${ }^{24}$ Since this kind of singularities usually appears when the mass of the external particle is very close to the threshold of intermediate states, it may change the threshold behavior dramatically and show up directly as a bump in the amplitude. ${ }^{25,26}$

The explicit values of the coupling constants are not well determined for the moment. In order to obtain some less model-dependent results, we will mainly pay attention to the line shape behavior of the pertinent invariant mass spectrum only.

\section{1. $Y(4260) \rightarrow \bar{D} D^{*} \pi$}

Taking AB in Fig. 1(b) as $\bar{D} D^{*}$, we will firstly discuss the $\bar{D} D^{*} \pi$ final states, and mainly concentrate on the lineshape behavior of $\bar{D} D^{*}$ invariant mass spectrum.

Concerning the $S$-wave rescattering process $\bar{D} D^{*} \rightarrow \bar{D} D^{*}$, according to the power counting schemes discussed in the Refs. 27, 28, the contact interactions will stay at the leading order while one pion exchange can be considered as a subleading order correction. By utilizing the effective contact interaction, we concentrate on the enhancement structure around $3.9 \mathrm{GeV}$ resulting from the cusp effect instead of a genuine state.

The $D_{1} \rightarrow D^{*} \pi$ is a $\mathrm{D}$-wave decay while the $S$-wave decay is forbidden according to the heavy quark spin symmetry, which is one of the main reasons why this $D_{1}$ is so narrow. For the process $Y(4260) \rightarrow \bar{D} D_{1} \rightarrow \bar{D} D^{*} \pi$, the angular momentum between the pion and $\bar{D} D^{*}$ system is a D-wave. The amplitude will be proportional to $\left|\mathbf{p}_{\pi}\right|^{2}$, where $\mathbf{p}_{\pi}$ is the three-momentum of pion meson. The partial width $d \Gamma / d M_{\bar{D} D^{*}}$ will be proportional to (and very sensitive to) $\left|\mathbf{p}_{\pi}\right|^{5}$. The threshold of the $\bar{D} D^{*}$ system exactly corresponds to $\left|\mathbf{p}_{\pi}\right|_{\max }$. Although only the tree diagram is considered, there appears an obvious threshold enhancement on the $\bar{D} D^{*}$ distribution, as illustrated in Fig. 2(a). Since $Y(4260)$ just stays in the vicinity of the $D_{1} \bar{D}$ threshold, the momenta of $\bar{D}$ and $D^{*}$ via $Y(4260) \rightarrow \bar{D} D_{1}$ decays are small. And from the above discussion it could be concluded that most of the $\bar{D} D^{*}$ events will be accumulated in the near threshold region, where we may expect strong FSIs. According to the above effective Lagrangian, the transition amplitude corresponding to the rescattering process Fig. 1(b) reads

$$
T_{Y \rightarrow \pi D^{*} \bar{D}}^{\operatorname{loop}_{i}}=i g_{c} \int \frac{d^{4} l}{(2 \pi)^{4}} \frac{3 p_{\pi} \cdot \epsilon_{Y} p_{\pi} \cdot \epsilon_{D^{*}}+\left(\left(v \cdot p_{\pi}\right)^{2}-p_{\pi}^{2}\right) \epsilon_{Y} \cdot \epsilon_{D^{*}}}{\left(l^{2}-m_{D}^{2}\right)\left((P-l)^{2}-m_{D_{1}}^{2}\right)\left(\left(P-p_{\pi}-l\right)^{2}-m_{D^{*}}^{2}\right)},
$$



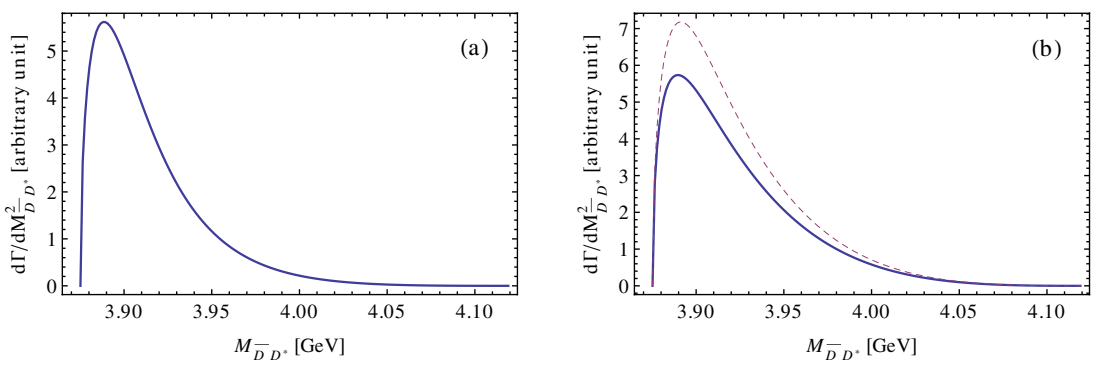

Fig. 2. Invariant mass spectrum of $\bar{D} D^{*}$ in $Y(4260) \rightarrow D_{1} \bar{D} \rightarrow \bar{D} D^{*} \pi$. (a) and (b) Correspond to tree and loop diagrams, respectively. In (b), the solid (dashed) line corresponds to the result with (without) taking into account the width of $D_{1}$.

where $g_{c}$ is the combination of some relevant coupling constants, $\epsilon_{Y}$ and $\epsilon_{D^{*}}$ are the polarization vectors of $Y(4260)$ and $D^{*}$ respectively, and $P$ is the momentum of the initial particle. The sums of the polarizations for the heavy vector mesons are $\sum \epsilon^{\mu} \epsilon^{* \nu}=-g^{\mu \nu}+v^{\mu} v^{\nu}$. In Eq. (2), there is no integral momentum appearing in the nominator. It is therefore just a scalar three point function, which can be taken as a form factor for the $Y \psi \pi \pi$ coupling. The two-cut condition indicates the singularity arising from the triangle diagram in the form factor coupling. The numerical result corresponding to the rescattering diagram is displayed in Fig. 2(b), where an obvious threshold enhancement is also observed. However, we have not taken into account the interference between the tree and loop diagram, since there are some unknown coupling constants. It is also not easy to judge quantitatively the relative strength between the loop and tree diagram for the moment. It should be mentioned that some other rescattering amplitudes share the similar formula with Eq. (2). For brevity, we will omit them in the following discussions.

The states $D_{0}$ and $D_{1}^{\prime}$ are too broad to be taken as the components of a relatively narrower molecular state. However, since the thresholds of $D_{0} \bar{D}^{*}$ and $D_{1}^{\prime} \bar{D}$ are very close to the mass of $Y(4260)$, it is still justifiable to assume larger couplings of $Y(4260)$ with these combinations. We will try to explore the line shape behaviors under such assumptions in the following. The threshold of $D_{2} \bar{D}$ is also close to $Y(4260)$, but its coupling is of $D$-wave, which should be suppressed compared with other combinations.

$D_{0} \rightarrow D \pi$ and $D_{1}^{\prime} \rightarrow D^{*} \pi$ are $S$-wave decays. Therefore only the tree diagram itself can not lead to obvious threshold enhancement structures, as illustrated in Figs. 3(a) and 4(a). When evaluating the loop diagram, since the intermediate states are very broad, we also need to take into account the influence of the larger width. For quantitatively estimating this influence, the propagator $l^{2}-m^{2}$ will be changed into $l^{2}-m^{2}+i m \Gamma$ in Eq. (2), where $\Gamma$ is the decay width of the corresponding state with mass $m$. From Figs. 3(b) and 4(b), we can see that it will lower the rescattering amplitude when considering the broad width influence. After taking into account the rescattering processes, it seems that the threshold enhancement behavior is 

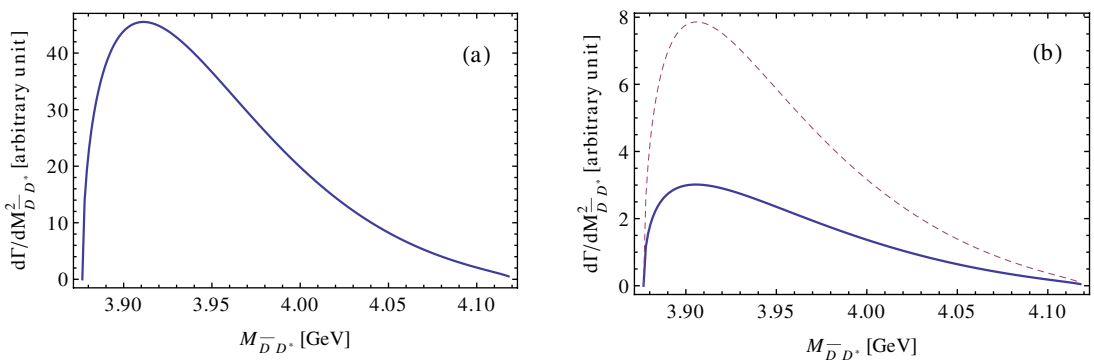

Fig. 3. Invariant mass spectrum of $\bar{D} D^{*}$ in $Y(4260) \rightarrow \bar{D}_{0} D^{*} \rightarrow \bar{D} D^{*} \pi$. (a) and (b) Correspond to tree and loop diagrams, respectively. In (b), the solid (dashed) line corresponds to the result with (without) taking into account the width of $D_{0}$.


Fig. 4. Invariant mass spectrum of $\bar{D} D^{*}$ in $Y(4260) \rightarrow D_{1}^{\prime} \bar{D} \rightarrow \bar{D} D^{*} \pi$. (a) and (b) Correspond to tree and loop diagrams, respectively. In (b), the solid (dashed) line corresponds to the result with (without) taking into account the width of $D_{1}^{\prime}$.

still not significant compared with the situation in the $Y(4260) \rightarrow D_{1} \bar{D} \rightarrow \bar{D} D^{*} \pi$ process, but they may offer some background when analyzing these decay channels.

\section{2. $Y(4260) \rightarrow J / \psi\left(\psi^{\prime}\right) \pi \pi$}

Taking AB appeared in Fig. 1(b) as $J / \psi\left(\psi^{\prime}\right) \pi$, we will discuss the $J / \psi\left(\psi^{\prime}\right) \pi \pi$ final states. As mentioned in the previous section, the relatively larger $D^{*} \bar{D}$ yield in the vicinity of threshold will favor strong FSIs. In combination with the TS that may appear in the rescattering amplitude, there will be a strong enhancement in $J / \psi \pi$ $\left(\psi^{\prime} \pi\right)$ invariant mass spectrum which lies at the $D^{*} \bar{D}$ threshold. As illustrated in Fig. 5, only according to this semi-quantitative estimate, the line shape behavior is already very similar with the results observed by BESIII and Belle, and the "width" of this cusp structure can be compared with the width of " $Z_{c}(3900)$ " to some extent. In $\psi^{\prime} \pi \pi$ final states, as the kinematics changed, the reflection of the narrow cusp, which behaves as a bump, has been shifted to the tail of the phase space.

We also investigate some other possible combinations which may produce similar cusp structures, such as the processes $Y(4260) \rightarrow D_{0} \bar{D}^{*}[D] \rightarrow J / \psi\left(\psi^{\prime}\right) \pi \pi$ and $Y(4260) \rightarrow D_{1}^{\prime} \bar{D}\left[D^{*}\right] \rightarrow J / \psi\left(\psi^{\prime}\right) \pi \pi$, where the charmed meson $D\left(D^{*}\right)$ in the bracket denotes the exchanging particles between $D_{0}$ and $\bar{D}^{*}\left(D_{1}^{\prime}\right.$ and $\left.\bar{D}\right)$. The 

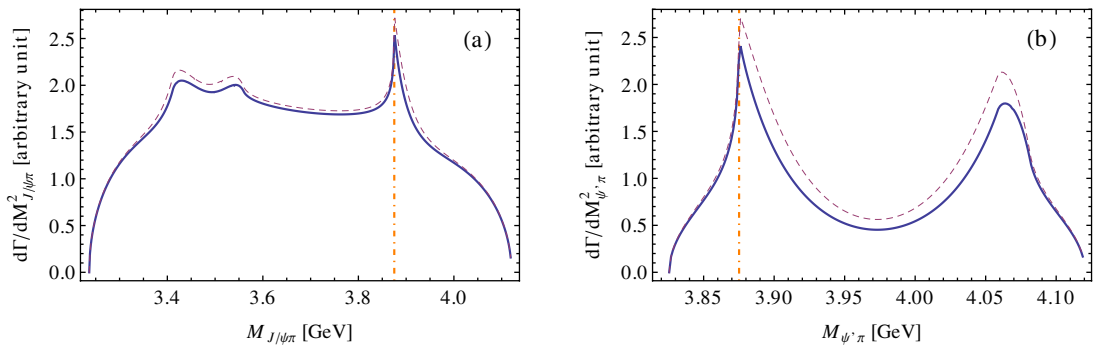

Fig. 5. Invariant mass spectrum of $J / \psi \pi\left(\psi^{\prime} \pi\right)$ in $Y(4260) \rightarrow D_{1} \bar{D}\left[D^{*}\right] \rightarrow J / \psi\left(\psi^{\prime}\right) \pi \pi$. Solid (dashed) line corresponds to the result with (without) taking into account the width of $D_{1}$. The vertical dot-dashed line indicates the threshold of $\bar{D} D^{*}$.
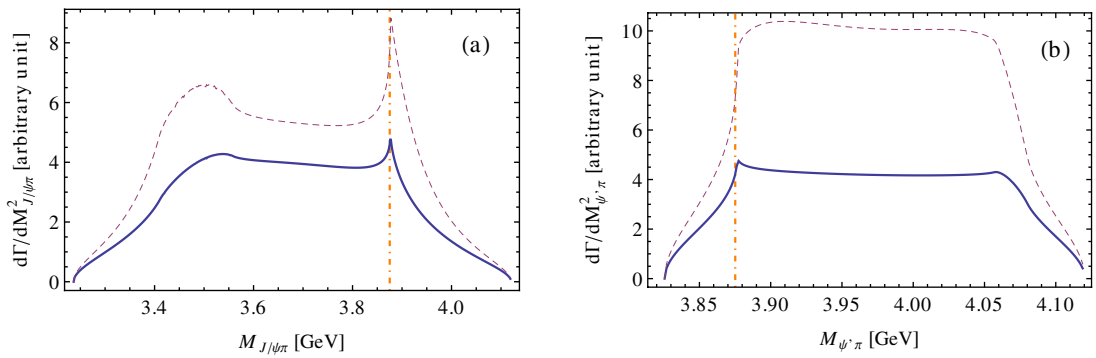

Fig. 6. Invariant mass spectrum of $J / \psi \pi\left(\psi^{\prime} \pi\right)$ in $Y(4260) \rightarrow D_{0} \bar{D}^{*}[D] \rightarrow J / \psi\left(\psi^{\prime}\right) \pi \pi$. Solid (dashed) line corresponds to the result with (without) taking into account the width of $D_{0}$. The vertical dot-dashed line indicates the threshold of $\bar{D} D^{*}$.
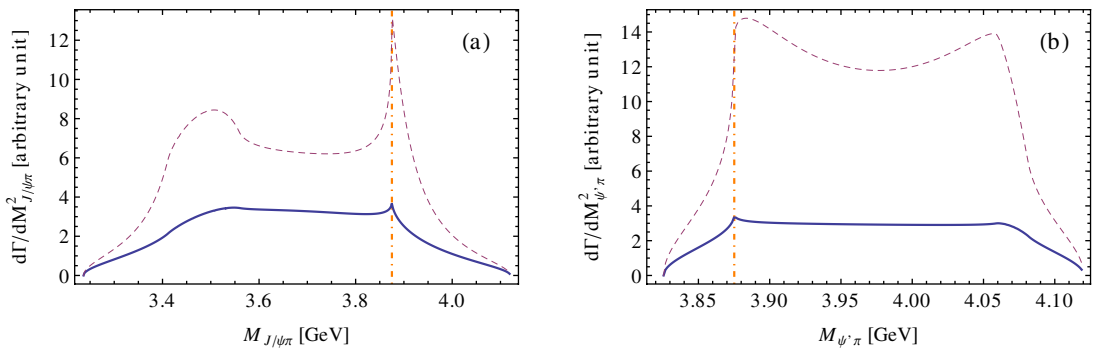

Fig. 7. Invariant mass spectrum of $J / \psi \pi\left(\psi^{\prime} \pi\right)$ in $Y(4260) \rightarrow D_{1}^{\prime} \bar{D}\left[D^{*}\right] \rightarrow J / \psi\left(\psi^{\prime}\right) \pi \pi$. Solid (dashed) line corresponds to the result with (without) taking into account the width of $D_{1}^{\prime}$. The vertical dot-dashed line indicates the threshold of $\bar{D} D^{*}$.

numerical results are displayed in Figs. 6 and 7. It seems that there will also be obvious cusp structures in the $J / \psi \pi \pi$ decay mode if the larger width of intermediate states is ignored. For the situation in $Y(4260) \rightarrow \bar{D} D^{*} \pi$, the broad width will lower the amplitude, and the cusp structure will be smoothed out to some extent. However if $Y(4260)$ has sizeable couplings with $D_{0} \bar{D}^{*}$ and $D_{1}^{\prime} \bar{D}$, these structures still could offer some background to the pertinent decay modes. The threshold of $\psi^{\prime} \pi$ is close to that of $\bar{D} D^{*}$. If comparing Figs. 6(b) or 7(b) with Fig. 5(b), it seems that only the TS itself cannot give an obvious cusp, since these distributions are 


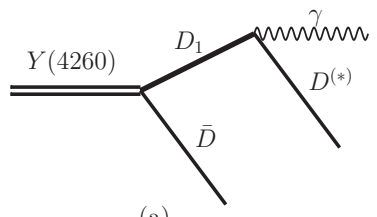

(a)

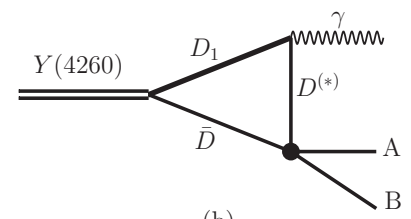

(b)

Fig. 8. (a): Radiative decays of $Y(4260)$ by supposing it is a $D_{1} \bar{D}$ molecular state. (b): the rescattering process, where $\mathrm{A}$ and $\mathrm{B}$ are some specified final states.
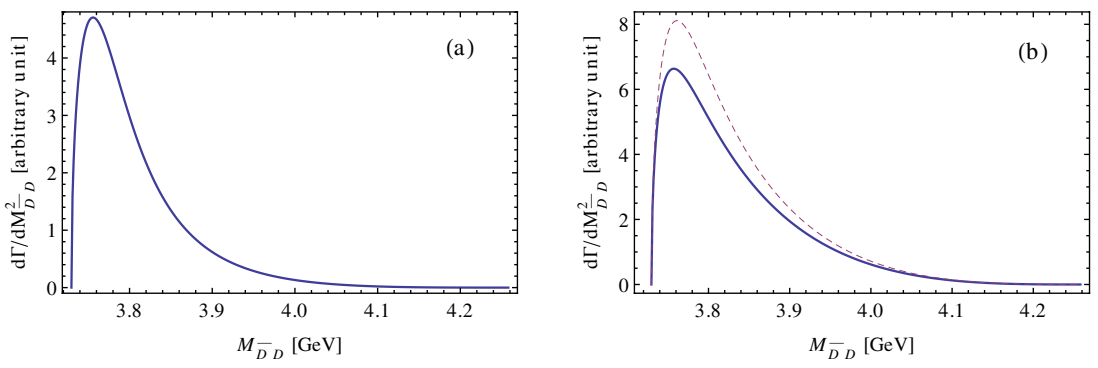

Fig. 9. Invariant mass spectrum of $\bar{D} D$ in $Y(4260) \rightarrow D_{1} \bar{D} \rightarrow \gamma D \bar{D}$. (a) and (b) correspond to the tree and loop diagrams respectively. Solid (dashed) line corresponds to the result with (without) taking into account the width of $D_{1}$.

results after integrating over phase space. Generating an obvious cusp also require more $\bar{D} D^{*}$ events should be produced at the threshold. The $D$-wave coupling of $D_{1} D^{*} \pi$ or introducing a resonance $Z_{c}(3900)$ can make it work. From this point of view, it seems that the $D_{1} \bar{D}$ combination is more favorable to be taken as the main component of $Y(4260)$. The $\psi^{\prime} \pi \pi$ decay mode could be used to test this argument.

\subsection{Threshold behavior in $Y(4260)$ radiative decays}

Radiative decay modes are very useful for understanding the intrinsic structures of hadrons. By assuming $Y(4260)$ is a $D_{1} \bar{D}$ molecule, we try to investigate some of its radiative decay properties under this scenario. As illustrated in Fig. 8, we will also concentrate on the threshold phenomena that results from the rescattering process. Since the kinematics is similar with the situation discussed in the previous sections, the TS is also expected to occur, which may strongly enhance the amplitude and change the corresponding line shape behavior. According to the results estimated by utilizing quark model, the radiative decay width of $D_{1}^{0} \rightarrow \gamma D^{(*) 0}$ is much larger than that of $D_{1}^{ \pm} \rightarrow \gamma D^{(*) \pm}$. This may imply that the ratio $R \equiv \Gamma\left(Y(4260) \rightarrow \gamma D^{(*) 0} \bar{D}^{0}\right) / \Gamma\left(Y(4260) \rightarrow \gamma D^{(*)+} D^{-}\right)$will be much larger than 1 , and the rescattering will mainly happen between neutral charmed anti-charmed meson pairs. However, if FSIs are strong, or if there is a larger branching ratio of $Y \rightarrow \gamma X \rightarrow \gamma D^{(*)} \bar{D}$, the ratio will be changed, where $X$ is some kind of charmonium state, such as $\chi_{c J}$ or $X(3872)$. 

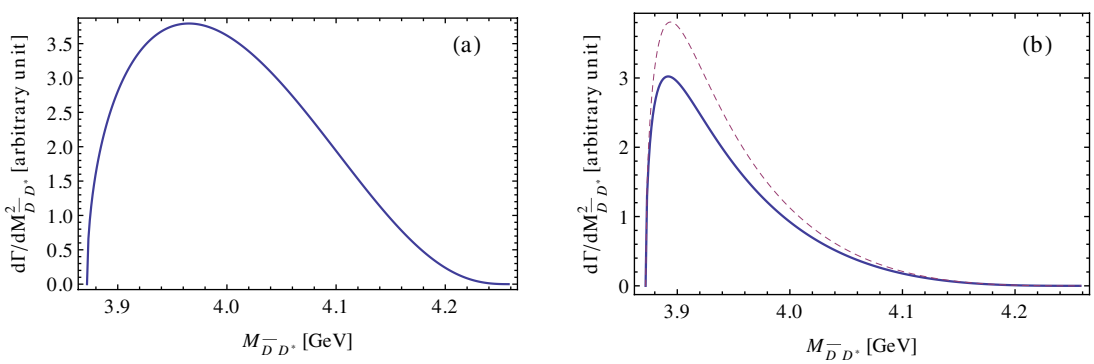

Fig. 10. Invariant mass spectrum of $\bar{D} D^{*}$ in $Y(4260) \rightarrow D_{1} \bar{D} \rightarrow \gamma D^{*} \bar{D}$. (a) and (b) Correspond to the tree and loop diagrams respectively. Solid (dashed) line corresponds to the result with (without) taking into account the width of $D_{1}$.
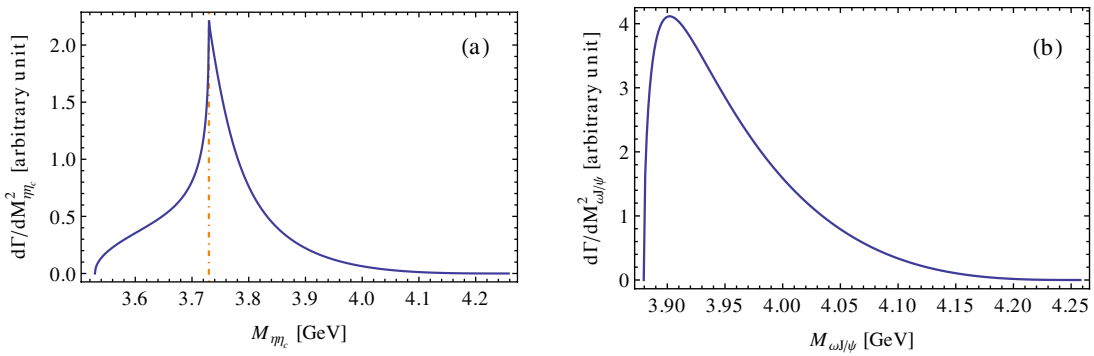

Fig. 11. (a) Invariant mass spectrum of $\eta_{c} \eta$ in $Y(4260) \rightarrow D_{1} \bar{D}[D] \rightarrow \gamma \eta_{c} \eta$. The vertical dot-dashed line corresponding to the threshold $2 m_{D}$. (b) Invariant mass spectrum of $J / \psi \omega$ in $Y(4260) \rightarrow D_{1} \bar{D}\left[D^{*}\right] \rightarrow \gamma J / \psi \omega$.

Taking the final states $\mathrm{AB}$ in Fig. 8 as $D \bar{D}$ and $D^{*} \bar{D}$ respectively, the numerical results without taking into account interference are displayed in Figs. 9 and 10. It can be noticed that in both of these two decay modes, the rescattering process can give obvious threshold enhancement. If taking $\mathrm{AB}$ as $\eta \eta_{c}$ and $\omega J / \psi$ respectively, and assuming an $S$-wave coupling, a narrow cusp structure is obtained at the $D \bar{D}$ as illustrated in Fig. 11(a). Since the threshold of $\omega J / \psi$ is a little higher than that of $D^{*} \bar{D}$, there is only a threshold enhancement at Fig. 11(b). These resonancelike structures do not result from some genuine resonances with quantum numbers $J^{P}=0^{+}$and $J^{P}=1^{+}$, but because of TS could occur in the rescattering amplitude, the narrow cusp structure is also expected. Especially for the narrow cusp structure in the vicinity of $D \bar{D}$ threshold, since there is no scalar charmonium state with the mass of about $2 m_{D}$ that has ever been observed, this radiative decay mode can be taken as a criterion to test whether such a structure is some kind of molecular state or just a cusp structure results from FSIs.

\section{Summary}

By assuming $Y(4260)$ is a $D_{1} \bar{D}$ molecular state, we investigate several decay modes which are related with this assumption. $\bar{D} D^{*} \pi$ will be the main decay channel 
under this ansatz, and since $D_{1} \rightarrow D^{*} \pi$ is $D$-wave decay, there will be more $\bar{D} D^{*}$ events accumulated in the vicinity of the threshold. Therefore, strong FSIs will be expected. With some special kinematic configurations, TS may occur in the transition amplitude, which will significantly change the threshold behavior and manifest itself as some cusp structures. The process $Y(4260) \rightarrow J / \psi\left(\psi^{\prime}\right) \pi \pi$ results from $\bar{D} D^{*}$ rescattering is investigated, where the cusp structure is very similar with the experimental observations. This is the result without introducing a genuine resonance, such as $Z_{c}(3900)$. However, we should also claim this effect just offer a possible dynamical mechanism to describe such a resonance-like structure. The existence of a genuine resonance is not excluded. And the other possible combinations $D_{0} \bar{D}^{*}$ and $D_{1}^{\prime} \bar{D}$, which are close to the mass of $Y(4260)$, are also taken into account. Although they are too broad to form a molecular state, they can also lead to some threshold enhancement structures, as long as they have sizeable couplings with the initial state. Some radiative decay modes of $Y(4260)$ under the molecule ansatz are also discussed, and we emphasize that the strong $D \bar{D} S$-wave interaction will lead to a narrow cusp structure in the process $Y(4260) \rightarrow D_{1} \bar{D}[D] \rightarrow \gamma \eta_{c} \eta$, which may behave as a scalar charmonium resonance.

\section{Acknowledgments}

This work was supported in part by China Postdoctoral Science Foundation under Grant No. 2013M530461, the National Natural Science Foundation of China under Grants No. 11275113, No. 11075004, No. 11021092 and No. 11261130311.

\section{References}

1. B. Aubert et al. [BaBar Collaboration], Phys. Rev. Lett. 95, 142001 (2005).

2. Q. He et al. [CLEO Collaboration], Phys. Rev. D 74, 091104 (2006).

3. C. Z. Yuan et al. [Belle Collaboration], Phys. Rev. Lett. 99, 182004 (2007).

4. G. Pakhlova et al. [Belle Collaboration], Phys. Rev. D 77, 011103 (2008).

5. B. Aubert et al. [BaBar Collaboration], Phys. Rev. D 79, 092001 (2009).

6. G. Pakhlova et al. [Belle Collaboration], Phys. Rev. D 80, 091101 (2009).

7. K. Abe et al. [Belle Collaboration], Phys. Rev. Lett. 98, 092001 (2007).

8. B. Aubert et al. [BaBar Collaboration], Phys. Rev. D 76, 111105 (2007).

9. M. Ablikim et al. [BES Collaboration], Phys. Lett. B 660, 315 (2008).

10. M. Ablikim et al. [ BESIII Collaboration], arXiv:1303.5949 [hep-ex].

11. Z. Q. Liu et al. [Belle Collaboration], arXiv:1304.0121 [hep-ex].

12. T. Xiao, S. Dobbs, A. Tomaradze and K. K. Seth, arXiv:1304.3036 [hep-ex].

13. S. K. Choi et al. [BELLE Collaboration], Phys. Rev. Lett. 100, 142001 (2008).

14. R. Mizuk et al. [Belle Collaboration], Phys. Rev. D 78, 072004 (2008).

15. A. Bondar et al. [Belle Collaboration], Phys. Rev. Lett. 108, 122001 (2012).

16. Q. Wang, C. Hanhart and Q. Zhao, arXiv:1303.6355 [hep-ph].

17. D.-Y. Chen, X. Liu and T. Matsuki, arXiv:1304.5845 [hep-ph].

18. D.-Y. Chen and X. Liu, Phys. Rev. D 84, 094003 (2011).

19. D.-Y. Chen, X. Liu and S.-L. Zhu, Phys. Rev. D 84, 074016 (2011).

20. D. V. Bugg, Europhys. Lett. 96, 11002 (2011).

21. D.-Y. Chen and X. Liu, Phys. Rev. D 84, 034032 (2011). 
22. X.-H. Liu and G. Li, Phys. Rev. D 88, 014013 (2013).

23. G. Li and X.-H. Liu, arXiv:1307.2622 [hep-ph].

24. J.-J. Wu, X.-H. Liu, Q. Zhao and B.-S. Zou, Phys. Rev. Lett. 108, 081803 (2012).

25. P. V. Landshoff and S. B. Treiman, Phys. Rev. 127, 649 (1962).

26. R. J. Eden, P. V. Landshoff, D. I. Olive and J. C. Polkinghorne, The Ananytic S-Matrix (Cambridge University Press, 1966).

27. M. T. AlFiky, F. Gabbiani and A. A. Petrov, Phys. Lett. B 640, 238 (2006).

28. M. P. Valderrama, Phys. Rev. D 85, 114037 (2012). 